P. I.-Very sudden onset with chills, sweating and temperature ranging between 103 and $105^{\circ} \mathrm{F}$. General physical cxamination negative except for the most marked tenderness I have ever seen over the kidney in a case of pyelitis in a child, so marked that perinephritic abscess was considered at once. The urine was filled with pus and blood, but with the free use of hexamethylenamin the condition quieted down in a weok. In four months she has had as many similar attacks much less severe. $\mathrm{As}$ it is, the child is in a state of invalidism and the family has objected to the more searching methods of diagnosis which Dr. Knight has proposed. Such a waste of time in making a correct diagnosis we feel to be entirely unwise.

In conclusion, a report of these infections by a medical man seems not inappropriate since practitioners are the ones who must first see them, and upon their promptness in making a diagnosis, based on a careful etiological survey, depends the outcome.

Cases of pyelitis of tuberculous origin should not be allowed to continue until the bladder is involved.

Renal infection must be considered in the differential diagnosis of every febrile affection.

The urine may show much or little according to the degree of intrarenal pressure and drainage and the condition of the ureter, so that frequent and thorough examinations of the sediment are necessary.

The two most constant signs are pus in the urine and some degree of tenderness in the kidney by bimanual palpation.

I desire to thank Dr. Arthur L. Chute for several suggestions and the other physicians mentioned for the privilege of reporting their rases.

\section{Oliniral Rppartment.}

\section{A CASE OF HUMAN RABIES.*}

By Harky Iinenthat, M.D., Moston,

Physician to Out-Patients, Mas8achusetts General Hospital; State Inspector of Health.

THe following case of rabies which was observed closely from the time the patient was bitten through the development and termination of the disease, presents many features of interest and a detailed report of it is justified. The patient received a full course of intensive Pasteur treatment without developing an immunity. Moreover, the quinine treatment which Harris ${ }^{1}$ reported to have cured a case, was tried without avail in this case, equally unavailable was a new method of treatment tried.

- I am indebted to Dr. C. Frothingham on whose service in the Peter Bent Brigham llospital the case was admitted and to Dra F. B. Grinnell and R. Kohn of the Department of Preventive Medicine of Harvard Medical School who followed the case with me.
M. C. N., twenty years of age, was bitten by a stray Scotch collie in the left thumb while walking in the street on the morning of July 25, 1914. The wound consisted of a slight longitudinal superficial abrasion of the distal phalanx of the left thumb and a punctured wound at the root of the nail into the matrix.

The dog did not, as far as could be learned, attempt to bite any one else but remained quietly on a near-by lawn till he was shot by a police officer about two hours later. $\mathrm{As}$ is unfortunately too often the case, the carcass was disposed of without an examination of the brain being made.

After being bittten the patient immediately reported to his family physician, who bathed the thumb for twenty minutes in $1: 1000$ corrosive sublimate solution and applied a corrosive dressing. The dressing was removed on July 27 and the wound was practically healed.

Several days later the case was brought to the attention of the State Department of Health by the family, who asked that Pasteur treatment be administered. Virus was obtained from the Hygienic Laboratory of the Public Health Service in Washington and on Aug. 1, seven days after the patient was bitten, the treatment was started.

The course of the treatment was uneventful with the following exceptions: On the seventh day of the treatment there was a very slight local reaction without constitutional disturbance at the site of the first injection. This cleared up in twenty-four hours. On the ninth day there was a slight local reaction at the site of the injection of the previous day. This reaction lasted less than twenty-four hours. On the thirteenth day of the treatment the patient complained of headache and nausea. The headache persisted for several days, during which time, however, his temperature remained normal. The treatment terminated on Aug. 21 and the patient was discharged, feeling in his usual good health. He was instructed to report from time to time as to his condition so that we might keep in close touch with him. He was cautioned to avoid any excessive physical exertion, to avoid exposure to cold and alcoholic beverages.

On the afternoon of Sept. 8, eighteen days after the completion of the Pasteur treatment and fortyfive days after he was bitten, he began to feel a numbness of his left thumb, which during the night extended up to his elbow; in addition to the numbness he experienced from time to time a sharp stinging pain shooting down his left forearm. An examination made on the morning of Sept. $\theta$ was entirely negative. He appeared in his usual good health, his deep and superficial reflexes were normal, and there were no sensory disturbances in his left thumb and forearm, the site of the numbness. The subjective feeling of numbness was much less than on the previous night. He reported that since the termination of the Pasteur treatment on Aug. 21 he felt well, his appetite was good, his bowels wero regular and he slept well. He was kept under observation all day but nothing developed. The next day the patient's mother reported by telephone that he had a sleepless night and that he was not feeling well generally. In order to keep him under constant observation and to have him near where he could be easily reached, arrangements were made with the Peter Bent Brigham Hospital, where he was admitted on the afternoon of Sept. 10. On admission he was found to have a slight elevation 
of temperature, otherwise the examination was entirely negative.

On Sept. 11 the numbness had diminished except when the thumb where he was bitten was pressed. His neck seemed a little stiff, but with distraction of patient's attention it could be easily moved. His reflexes were normal. There were no Kernig and no other signs suggestive of meningeal irritation. Heart showed a slight respiratory arrythmia. Slight elevation of his temperature persisted.

His urine was straw colored, acid in reaction, 1.009 specific gravity and except for a slightest possible trace of albumen was negative.

An examination of his blood showed a white count of 14,000 , of which $57 \%$ were polynuclears, $26 \%$ large mononuclears, $17 \%$ small mononuclears, erythrocytes not remarkable.

On the morning of Sept. 12 the patient complained that his ears were particularly sensitive to sounds. He was distressed by the ordinary sounds in the ward so that he had to place pieces of cotton in his ears. He also complained of double vision. Examination showed marked diplopia when focusing on an object two feet away; marked nystagmus particularly when eyes turned to right. Paresis of right external rectus. All superficial and deep reflexes were present. The plantar was present on the right, on the left the normal plantar was absent but there was a contraction of the fascia lata when the sole was stimulated. There were no Babinski, Oppenheim or Gordon reflexes, no Kernig and no neck sign.

At 1.30 in the afternoon of Sept. 12, the patient had a moderate chill, there was a marked general hyperaesthesia with increased response to stimuli of all kinds. Photophobia became extreme. There was dilatation of the right pupil though it reacted to light. The muscles of the eyelids twitched continually and there were also occusional slight twitchings of the muscles of the arms, more marked on the right side. He complained of a choking sensation and began to raise large quantities of saliva. His respiration became jerky and sobbing in character and spasms of the muscles of deglutition became pronounced. He would make quite elaborate preparations before taking a drink, such as clearing his throat, changing position, etc., he would then take the water in his mouth and hold it there for several seconds; then ho would make a sudden effort to swallow it, bringing on severe spasms. He was given a gram of chloral and fifteen mgms. of morphia, which quieted him somewhat.

His mind was perfectly clear during the afternoon; towards evening he became mildly delirious, was rather talkative, though well oriented.

At eight o'clock Dr. I. C. Walker administered 14 c.c. of salvarsanized serum intraspinally. Fifteen minutes later he was given $1 \mathrm{gm}$. of quinine and urea hydrochlorate intravenously. There was an immediate violent reaction to the intravenous injection, the patient's face became flushed and dusky, he broke out in a profuse perspiration and within one minute after the injection he complained of intense buzzing in his ears. His pulse became somewhat accelerated and he was distinctly uncomfortable. His condition steadily grew worse, so that in view of the intense reaction to the quinine no more was administered. About midnight he became noisy, delirious and irrational and was restrained in bed with diffculty. He had hallucinations of dogs chasing him. Several doses of morphia and atropine were given to him with little effect. The patient gradually be- came weaker and died at 4.20 a.m. on Sept. 13 of respiratory failure.

The spinal fluid, 25 c.c. of which was withdrawn at the time the intraspinous injection was given, was slightly cloudy, forming a delicate filamentous clot on standing. There were 600 cells per $\mathrm{cmm}$. of which $40 \%$ wero polymorphonuclears, $20 \%$ ' small lymphocytes, $38 \%$ large lymphocytes and $1 \%$ endothelials. No plasma cells. Wassermann negative. Dr. F. 13. Grinnell injected $1 / 2$ c.c. of the fluid subdurally into each of four rabbits; none of them came down with the disease.

\section{MORTALITY FROM RABIES.}

The incidence of rabies from bites of rabid animals as compiled in different statistics shows considerable variation. It depends upon the age of the person bitten, the severity of the injury and the location of the bite, the nearer the head the more likelihood of the development of the disease. From the statistics compiled by various writers it would seem that about $16.20 \%$ of persons bitten by rabid animals develop the disease.

Once the symptoms of the disease develop the termination is almost invariably fatal. Some aborted types of the disease have been reported as well as recovery after symptoms have developed, but the diagnoses in such cases have been questioned. It is safe to state that if recovery occurs the disease was almost to a certainty not rabies.

Pasteur treatment has reduced the mortality from the bites of rabid animals to about $1 \%$. Not all deaths from rabies among those taking the Pasteur treatment, however, can be regarded as due to the failure of the treatment. The establishment of an immunity to rabies by the injection of attenuated fixed virus is a slow process requiring three weeks. Fortunately the incubation period of rabies is usually a long one so that there is sufficient time to estabish an immunity. Some cases, however, have a short incubation period, and the disease develops before an immunity can be established. Such cases cannot be regarded as failure of the treatment. That certain individuals, however, will fail to develop an immunity by the treatment is shown by the occasional development of the disease long after the treatment has been completed.

In this case eighteen days elapsed between the completion of the treatment and the onset of the symptoms, a time sufficiently long to establish an immunity if the treatment were effective. This case must be regarded as one of the rare failures of the Pasteur treatment.

\section{TREATMENT OF RABIES,}

In $1913 \mathrm{Moon}^{2}$ published experimental results with the use of quinine in the treatment of rabies in dogs. Dogs to whom quinine sulphate was administered recovered from rabies while those who did not get the treatment died from the disease. 
In October of the same year Harris 1 reported a case of human rabies which recovered after the intravenous administration of quinine and urea hydrochlorate. The experimental results of Moon have not been confirmed by Frothingham and IIolliday and by Cummings. Nor has quinine proved efficacious in two cases of human rabics reported since the publication of Harris's report. The case reported here must be added to the other cases unsuccessfully treated with quinine.

Salvarsan and neosalvarsan have been used unsuccessfully in the treatment of the disease. $\Lambda \mathrm{s}$ far as I can find, this was the first time salvarsanized scrum was used intraspinally, but unfortunately with no success.

\section{PUBLIC HEALTI ASPECT OF RABIES.}

I have had the opportunity to see two cases of human rabies within a few months. The case not here reported I saw in the terminal stage of the disease. The patient, a man of 45 , was bitten by a dog six weeks previous to the development of the symptoms. The dog was killed by a police officer and the carcass disposed of without an examination being made. No Pasteur treatment was given.

To see a case of human rabies is sufficient to make one wish never to have to see another one. 'The pity of it all is that this most horrible of diseases is preventable. What can be accomplished by preventive measures can be seen by what has been achieved in Australia and England, where by the simple muzzling of dogs the discase was entirely eradicated.

In the nine-year period 1905-1913 inclusive, twenty-five deaths from rabies have been reported in Masssachusetts. We must assume the responsibility for the inexpressible physical and mental suffering of those afflicted, for the agonizing states of mind of the families and friends of the victims, and for the anguish and anxiety of those bitten who undergo the treatment. This dreadful disease exists with our consent and its victims die with our approval.

REYERENCEN.

1 Journal American Medical Association, Oct. 25, 1013.

a Journal Inf. Die. July, 1013.

s Journal Medical Reevarch, Vol, xxx, 1014.

- Journal Inf. Dis. Vol, xv, 1014.

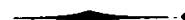

\section{fllpdiral pronreas.}

\section{REPOR'T OF PROGRESS IN MENTAL DISEASES.}

PART I.

By Henry R. Stedman, M.D., Brookune, Mass.

PARAPHRENIA.

IINDER this head Kraepelin ${ }^{1}$ separates from dementia precox a new group, still employing the criterion of the final outcome. He says that in dementia precox the disorganization of the psychic personality affects chiefly the affective life and will. The paraphrenic group, a small one, in spite of manifold points of accord with the manifestations of dementia precox yet has far less influence upon the affects and will, and consequently results in much less disturbance of the inner character of mental life. The main disturbance here is with the intellectual functions. These patients even in the last stages do not show the affective dulness and indifference which represent so frequently even the earliest signs of dementia precox. The actions and conduct are in the essence affected only by the abnormal trend of thought and mood. "Independent disturbances of the will," which tend to accompany dementia precox in such manifold form (c.g., mannerisms, impulsive acts, stereotypies, etc.), occur here only exceptionally.

(1) Paraphrenia Systematica, characterized by the insidious onset of steadily growing delusions of persecution, with later ideas of grandeur but without destruction of personality. The disease takes years to develop; ideas of reference and sense-falsifications play a considerable part in its evolution. The delusions are well systematized and may be accompanied by memory falsification. There scems to be no end-stage as the disorder continues progressively throughout life. Recovery does not occur, but it is especially emphasized that there is no loss of interest.

The close resemblance of this picture to that described by others under the title of paranoia needs but little emphasis. In speaking of the relation of paranoia to dementia precox Kraepelin claims that there can be no difficulty in distinguishing between the two because of the absence in the former of any evidence of indepen dent disturbances of will or of signs of affective deterioration. This distinction seems hardly to hold good with regard to paraphrenia systematica and we must await the appearance of the volume dealing with paranoia for enlightenment.

(2) The expansive type characterized by the rich development of grandiose ideas, generally with heightened mood and some slight excitement. This is an extremely interesting, though small, group composed almost entirely of women, and the cases have hitherto been usually classed with the manic-depressive disorders, as was done by Kraepelin himself, or with paranoia. The disorder is of slow progressive evolution and like the last, reaches no end-stage and presents little or no loss of interest.

(3) Confabulating form, very similar to the last, but characterized by the development of very marked memory falsifications.

(4) Paraphrenia Phantastica. Under this name are included the small group of cases originally classed as dementia paranoids. They are characterized by the development of the most phantastic and incoherent delusions. Beliefs are elaborated and maintained which seem to surpass 